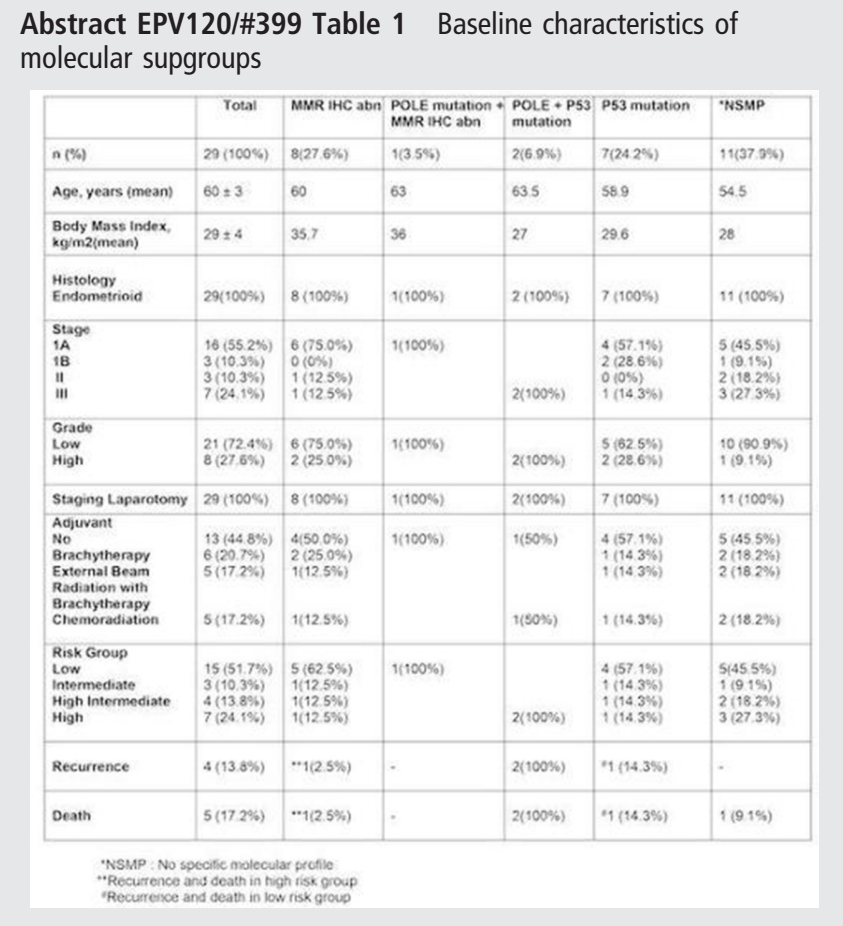

Results Results/Conclusions An interim analysis of 29 patients was done. Eight $(27.6 \%)$ patients had MLH1 mutation, 1 (3.5\%) patient had POLE and MLH1 mutation, while 2 (6.9\%) had both POLE and P53 mutation. Seven (24.2\%) patients were found to have null mutations of P53, while the remaining 11 (37.9\%) had no specific molecular profile (NSMP). ESMO-ESGO risk group correlation, recurrences, and deaths are shown in table 1 .

Conclusions Implications: Recurrence in low risk groups, behaviour of multiple classifiers, NSMP group and POLE mutated higher risk/stage cancers are areas still underresearched. A larger study exploring the integrated approach will help answer these questions and open novel avenue of research aimed at immunotherapy in endometrial cancer especially in recurrent settings.

\section{EPV121/\#419 BASELINE CLINICAL OUTCOMES OF LYNCH SYNDROME PATIENTS UNDERGOING ANNUAL SURVEILLANCE VERSUS RISK-REDUCING SURGERY IN A PROSPECTIVE COHORT STUDY}

${ }^{1} \mathrm{~S}$ Piedimonte*, ${ }^{2} \mathrm{~A}$ Zia, ${ }^{3} \mathrm{M}$ Maganti, ${ }^{2} \mathrm{~S}$ Ferguson. 'University of Toronto, Gynecologic Oncolgoy, Toronto, Canada; ${ }^{2}$ Princess Margaret Cancer Centre/University of Health Network/Sinai Health Systems, Gynecologic Oncology, Toronto, Canada; ${ }^{3}$ Princess Margaret Cancer Centre, Biostatistics, Toronto, Canada

\subsection{6/ijgc-2021-IGCS.191}

Objectives To describe baseline characteristics of Lynch syndrome(LS) patients enrolled in a prospective study of annual surveillance versus risk-reducing surgery(RRS) and determine prevalent cases of endometrial intraepithelial neoplasia(EIN), endometrial(EC) and ovarian(OC) cancers

Methods A prospective cohort study was implemented in February 2015 for LS patients diagnosed based on a pathogenic variant in mismatch repair genes but unaffected by gynecologic cancer. Baseline investigations included CA-125, ultrasound and endometrial biopsy(EMB); further investigations were performed as warranted. Patients were recommended RRS by age 40 or following child-bearing. All others had annual surveillance and analyzed per treatment received.

Results Among 82 patients, 41 underwent RRS and 41 annual surveillance. The most frequent mutation was MSH6(34.1\%). $25.9 \%$ had a personal history of LS-associated cancer and 97.5\% had a family history, most commonly being colorectal (74.4\%). Patients in the RRS group had a higher median age at LS diagnosis(47 vs 32 years, $p<0.001)$ and entry into LS screening program(47 vs 33 years, $\mathrm{p}<0.001)$. At baseline, median CA-125 was 10 in both groups $(\mathrm{p}=0.65)$. The baseline EMB rate was $85 \%(n=70)$ with an abnormality rate of $4.88 \%$ (two EIN in surveillance group and one EC in RRS group). Seventy(91\%) individuals underwent baseline ultrasound and no OCs were detected. In patients undergoing RRS, the median time from initial visit to surgery was 6.1 months(range 1.1-20.7); 3 additional EINs were diagnosed on final pathology.

Conclusions In LS patients followed in a surveillance program, the prevalent rate of EIN/EC is $5-10 \%$, mostly in the RRS group. RRS within the recommended time prevents diagnosis of significant pathology.

\section{EPV122/\#421 ISOLATED LYMPHATIC RECURRENCE IN ENDOMETRIAL CANCER: A RETROSPECTIVE STUDY}

${ }^{1} \mathrm{~S}$ Garzon, ${ }^{1} \mathrm{~S}$ Kumar, ${ }^{2} \mathrm{~A}$ Weaver, ${ }^{2} \mathrm{M}$ Mcgree, ${ }^{3} \mathrm{~S}$ Uccella, ${ }^{4} \mathrm{G}$ Keeney, ${ }^{1} \mathrm{~W}$ Cliby, ${ }^{1} \mathrm{~A}$ Mariani. ${ }^{1}$ Mayo Clinic, Department of Obstetrics and Gynecology, Rochester, USA; ${ }^{2}$ Division of Biomedical Statistics and Informatics, Department of Health Sciences Research, Rochester, USA; ${ }^{3}$ AOUI Verona, University of Verona, Department of Obstetrics and Gynecology, Verona, Italy; ${ }^{4}$ Mayo Clinic, Department of Laboratory Medicine and Pathology, Rochester, USA

\subsection{6/ijgc-2021-IGCS.192}

Objectives We investigated factors associated with cause-specific survival (CSS) after isolated lymphatic recurrence (ILR) in endometrial cancer (EC).

Methods We identified patients who developed ILR among 4,216 EC patients surgically treated at the Mayo Clinic between 1984 and 2017. ILR was defined as the first and unique evidence of recurrence in lymph node-bearing areas (with or without $( \pm)$ vaginal recurrence). Univariate and multivariable Cox regression analysis was used to evaluate factors associated with CSS after ILR.

Results We observed 70 cases of ILR: 12 pelvic, 15 paraaortic, 14 pelvic and paraaortic, and 29 distant ( \pm pelvic and/or paraaortic). Most women (90.0\%) underwent pelvic and/or paraaortic lymphadenectomy during primary surgery, and $68.3 \%$ had positive nodes. Among 70 patients, 50 died of disease with median survival after ILR of 1.4 years. Patients who did not die of EC had a median follow-up after ILR of 6.6 (IQR 4.8-10.0) years. By univariate analysis, histologic grade, lymphovascular space invasion, ILR site, concomitant vaginal recurrence, and ILR treatment were significantly associated with CSS after ILR. CSS after ILR was not associated with primary lymphadenectomy, stage, or adjuvant therapy. Results of the multivariable analysis are reported in the Table.

Conclusions Histologic grade 2 or 3 of the primary tumor and concomitant recurrence in the pelvic and paraaortic lymph node basins or at the vaginal cuff were independent 